\title{
German centre set to improve brain's image
}

\section{Alison Abbott, Tübingen}

The Max Planck Society (MPS) has pledged to support a major new facility for developing neural-imaging technology at Tübingen.

The centre, which could be up and running as early as 2003 , will help to develop invasive and non-invasive functional imaging techniques. The MPS plans to invest up to DM60 million (US\$28 million) in the facility, which could become one of the world's leading centres of excellence in the fast-expanding field of brain imaging.

Brain-imaging techniques, including functional magnetic resonance imaging (fMRI), allow scientists to look at neurological function in living brains. But at the moment they do this indirectly, making use of magnetic effects to track the flow of oxygenated haemoglobin and so allowing researchers to deduce neural activity.

However, the Greek imaging specialist Nikos Logothetis, who will work at the new centre, has made considerable progress recently in developing ways to observe neural activity more accurately. Neuroscientists are optimistic that, with more powerful fMRI machines and further research into their use, they will rapidly advance the usefulness of the technique.

\section{Head start}

The decision by the MPS to fund the new centre so generously seems to be partly motivated by its desire to keep Logothetis in Germany. He was hired from Baylor College of Medicine in Houston, Texas, to become a director of the Max Planck Institute for Biological Cybernetics in Tübingen in 1996.

At the moment, the institute uses two magnetic-resonance machines, with maximum magnetic fields of 4.7 and 7.0 tesla, to analyse monkey brains.

As part of its work, Logothetis has helped to establish how the signal detected by the machines relates to neural activity.

Magnetic-resonance imaging - or nuclear magnetic resonance, as it used to be called - is widely used by doctors for anatomical imaging. It is also increasingly used experimentally for imaging the brain at work. But scientists are still learning how to interpret the images, which reflect the distribution of oxygen in the brain.

Researchers generally assume that high oxygen consumption reflects high levels of neural activity. But the new techniques developed by Logothetis use electrodes to monitor neural activity in monkey brains directly, while also using fMRI.

His most recent paper shows that fMRI signals correlate "unexpectedly well" with neuronal signals (see Nature 412, 150-157;

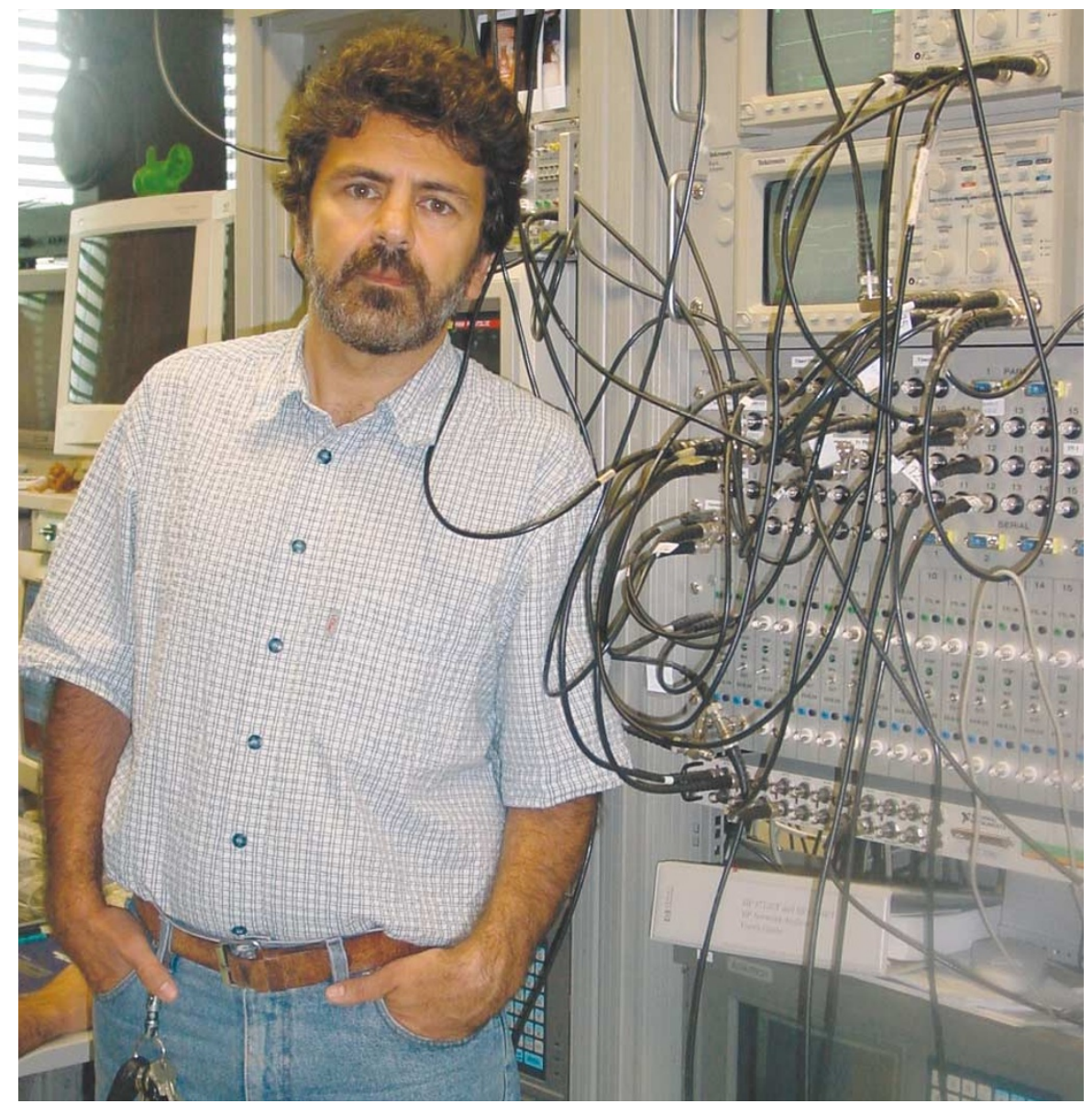

Wired up: Nikos Logothetis plans to track the control and release of neural signals in the brain.

2001), but that measured signals routinely underestimate the extent of neuronal activity.

"The haemodynamic (vascular) signal is an important tool," says Logothetis, "but the neuronal signal is more robust."

He now wants to develop new technologies for neural imaging that measure the neural signal directly rather than through a surrogate.

Logothetis hopes to make use of newly emerging in vivo techniques for non-invasive monitoring of single molecules (see Nature 412,372-374; 2001). "These new techniques are just waiting to be applied to questions interesting for neuroscientists," he says.

He plans, for example, to develop ways to track the calcium flow that triggers neural signals and to follow neurotransmitter release. This will require work by skilled physicists and chemists, as well as magneticresonance machines that generate strong magnetic fields. The MPS says it will provide these at the new centre.

The society is currently seeking a director with experience in this 'high-field' magnetic resonance to develop and run the centre. Logothetis has pledged to stay at Tübingen.

"The prospect of having access to such a centre on the campus where I work was fundamental to my decision," he says.

The centre will include a range of highfield fMRI machines, including very highfield machines for developmental work. A machine that generates a field of 15-16 tesla will be procured for work on small animals, and one of 7.0-9.5 tesla will be used for work involving humans.

\section{Redrawing the map}

The MPS hopes that Tübingen's home state of Baden-Württemberg, as well as nearby universities, will help to extend the centre. The state government has already said that it is interested in participating.

"Proposing a 15-tesla machine is a very bold move," says Robert Turner, a neuroimaging expert at University College London's Institute of Neurology. "The creation of the centre will change the map as far as imaging is concerned," he adds.

Marcus Raichle, whose laboratory at Washington University in St Louis, Missouri, pioneered the use of positron-emission tomography for non-invasive imaging, says that Logothetis's laboratory "has made enormous technical progress" in the past few years. Its expansion plan is "extremely exciting", says Raichle. 